\title{
THE MODIFIED PARSEVAL EQUALITY OF STURM-LIOUVILLE PROBLEMS WITH COUPLED BOUNDARY CONDITION
}

\author{
Mu Dan, Jiong Sun, Ji-Jun Ao And Junhui XiE
}

\begin{abstract}
We consider the Sturm-Liouville(S-L) problems with coupled boundary condition and transmission condition. Defining a Hilbert space related to the transmission conditions, we discuss the S-L problems in this modified Hilbert space. We prove the Parseval equality of the S-L problems with the transmission conditions in a modified Hilbert space and derive the Green's function for these problems.
\end{abstract}

Mathematics subject classification (2010): 34L20, 47E05.

Keywords and phrases: Sturm-Liouville problems, Green's function, Parseval equality.

\section{REFERENCES}

[1] R. A. Adams And J. J. F. Fournier, Sobolev Spaces, Volume 140, Second Edition (Pure and Applied Mathematics), 2003.

[2] R. KH. Amirov, Eigenvalues and normalized eigenfunctions of discontinuous Sturm-Liouville problem with transmission conditions, J. Math. Anal. Appl. 317 (1) (2006), 163-176.

[3] P. B. BAiley, W. N. EveritT, AND A. ZetTL, Regular and singular Sturm-Liouville problems with coupled boundary conditions, Proc. Roy. Soc. Edinburgh (A) 126 (1996), 505-514.

[4] E. Bairamov, E. Ugurlu, The determinants of dissipative Sturm-Liouville operators with transmission conditions, Mathematical and Computer Modelling 53 (5-6) 2011, 805-813.

[5] Mu Dan, Jiong Sun, JiJun Ao, Asymptotic behaviour of eigenvalues and eigenfunctions of SturmLiouville problems with coupled boundary condition and transmission condition, Operators and Matrices 9 (4) 2015, 877-890.

[6] W. N. EVERITT, D. RACE, On necessary and sufficient conditions for the existence of Caratheodory solutions of ordinary differential equations, Quaest. Math. 3 (1976), 507-512.

[7] W. N. EveritT, G. NASRI-ROUDSARI, Sturm-Liouville problems with coupled boundary conditions and Lagrange interpolation series, Journal of Computational Analysis and Applications 1 (4) (1999), 319-347.

[8] M. Kobayashi, Comments on eigenfunction expansions of discontinuous Sturm-Liouville systems, Applied Mathematics Letters 2 (3) (1989), 239-241.

[9] M. A. NAIMARK, Linear Differential Operators, English transl. in: Ungar, New York (1968).

[10] C. SHIEH, V. A. YURKO, Inverse nodal and inverse spectral problems for discontinuous boundary value problems, J. Math. Anal. Appl. 347 (2008), 266-272.

[11] E. C. Titchmarsh, Eigenfunction Expansions Associated with Second-order Differential Equations, Clarendon Press, Oxford (1946).

[12] J. Weidmann, Linear Operators in Hilbert Spaces: Graduate texts in mathematics 68, SpringerVerlag, New York, 1980. translated by Joseph Szücs.

[13] C. YANG, Inverse nodal problems of discontinuous Sturm-Liouville operator, J. Differential Equations 254 (2013), 1992-2014.

[14] A. ZettL, Sturm-Liouville Theory, AMS, Mathematical Surveys and Monographs vol. 121 (2005). 